\title{
7 Monitoring Responsible Research and Innovation in the European research area The MoRRI project
}

Ingeborg Meijer and Wouter van de Klippe

\subsection{Introduction}

Responsible Research and Innovation (RRI) as a policy initiative exists to more closely align research and innovation (R\&I) processes and products with societal needs. RRI draws on a diversity of theoretical work which has in the past sought for ways to more intentionally incorporate societal reflection within R\&I processes (Stilgoe, Owen, \& Macnaghten, 2013). While drawing on a rich history of efforts to develop more open and democratized R\&I systems (for a discussion on the historical context of the discourse of RRI, see Rip, 2014), the explicit operationalization of RRI within European Commission (EC) R\&I policy can be traced back to the 2014 Rome Declaration of RRI.

The 2014 Rome Declaration of RRI described the concept as "the ongoing process of aligning research and innovation to the values, needs and expectations of society" (Italian Presidency of the Council of the European Union, 2014). Within the declaration, there were calls for the integration of RRI within R\&I policy, which have since been followed. The EC currently has an operational definition of RRI which draws on a diversity of theoretical elaboration (Owen, Macnaghten, \& Stilgoe, 2012; Pandza \& Ellwood, 2013; Stilgoe, Owen, \& Macnaghten, 2013; von Schomberg, 2013; Rip, 2014), and is as follows:

a process where all societal actors (researchers, citizens, policy makers, business representatives, third sector organisation representatives, etc.) work together during the whole R\&I process in order to better align R\&I outcomes with the values, needs and expectations of European society.

This operational definition of RRI contains six guiding "keys" of RRI, including: gender equality, science literacy and science education, public engagement, ethics, open access and governance. The aspirations of RRI are bold and will likely benefit from the development of tools which will further 
support the creation, evaluation, implementation, and adaptation of R\&I policy to accomplish these goals (Owen, Macnaghten, and Stilgoe, 2012).

The Monitoring the Evolution and Benefits of Responsible Research and Innovation (MoRRI) project (2014-2018) was the first large-scale effort to develop, implement, and assess an RRI monitoring system for the European research area (ERA). MoRRI was a three-and-a-half-year-long project which consisted of initial conceptual work, data collection, data analysis, reflections, adaptations, and findings. In order to identify and measure both the scope, benefits, and pitfalls of RRI within the ERA, the MoRRI project developed and operationalized a conceptual framework and methodology according to the EC's current definition and framework of RRI. Subsequently, the project tested this methodology for its ability to monitor the current state of RRI, based around various metrics which were developed and elaborated throughout the project. Finally, MoRRI conducted various case studies to assess the realized socio-economic and democratic impacts that the implementation of RRI might have had.

The monitoring system developed by the MoRRI project (Peter et al., 2018) consisted of $36+$ indicators which were based on a review of literature which covered the EC's six key areas of RRI. The process of developing this monitoring system included examining existing data sources, analysing their appropriateness, locating any potential gaps in coverage, and the iterative development of new indicators which required primary data collection. Throughout this chapter, primary data will refer to the collecting of new data through tools (surveys, questionnaires, etc.) that were created throughout the MoRRI project.

After the various indicators were developed and potential shortcomings were identified, an extensive data collection process covering all European Union (EU) Member States was then carried out. This data collection process consisted of a large-scale survey-based data collection, a set of case studies critically examining the effects of the implementation of RRI, collection and analysis of bibliometric and patent databases, and secondary analyses of existing data at both individual- and country-level data. The survey was distributed among researchers at European institutions, research-funding organizations (RFOs), research-performing organizations (RPOs), societal stakeholder organizations, and representatives in industry.

In this chapter, we continue with a description of the challenges of the MoRRI project (Section 7.1.2), describe the EC's six RRI keys (Section 7.1.3), discuss the development of the indicators (Section 7.1.4), highlight emerging patterns at the country level (Section 7.1.5), and discuss the identification of benefits of RRI (Section 7.1.6) and the way forward (Section 7.1.7).

\subsection{Challenges}

The task of creating a monitoring and evaluation system for the implementation of RRI and its benefits can be understood through the lenses of being a technical, scientific, practical, and irreducibly political challenge. 
- The challenge is technical: The growth of quantitative measures to control and evaluate a diversity of swathes of life is so pernicious and widespread that it has been dubbed a "veritable metric tide" (Wilsdon, Doubleday, \& Stirling, 2015). Indicators to assess countries, organizations, institutions, and even individuals within R\&I landscapes have become ubiquitous and controversial. Despite this growth of indicator-led life, few scholars have attempted to develop indicators of RRI, and even fewer have gone beyond the theoretical step of their creation and sought to populate these indicators with data. While some laudable efforts for the creation of such indicators have been developed (Wickson \& Carew, 2014; Strand \& Kaiser, 2015; Heras \& RuizMallén, 2017; Monsonís-Payá et al., 2017), each shows considerable (and understandable) humility towards the task. An emphasis on the need for a diversity of context-sensitive indicators has been repeatedly articulated. This importance was not lost throughout the MoRRI project, as it is vital to ensure that the creation of these indicators does not serve to foreclose potential notions of responsibility.

- The challenge is scientific: There exist gaps in knowledge in the efficacy of RRI policies, the practices that they ostensibly support, and the benefits that these aim to have. The MoRRI project sought to remedy these gaps in knowledge through the development of a theoretical frame to understand the links between policy, practice, and effect, and additionally provided empirical work to support this theory. This required an integration of work being conducted in various disciplinary contexts; most importantly, theoretical work elaborating RRI and quantitative social science work on monitoring and evaluation.

- The challenge is practical and political: Throughout the MoRRI project, there existed a myriad of technical and theoretical decisions. These choices, made according to a variety of different value systems which were continuously negotiated throughout the project, inevitably have impacts on what vision of RRI is monitored, evaluated, and supported. The successor project of SUPER MoRRI (acronym for Scientific Understanding and Provision of an Enhanced and Robust Monitoring system for RRI) will provide RFOs, RPOs, and other parties with tools and knowledge which may be needed for their own assessments and action plans for the implementation of RRI. This novel framing of a monitoring and evaluation system is to be thought of as an opportunity to facilitate change rather than a system of domination and control. Indeed, consortia members caution against RRI (and the evaluation and monitoring systems seeking to aid in its implementation) becoming a "box-ticking" exercise or bureaucratic hurdle for researchers and innovators. Proponents of RRI envision RRI to be a novel opportunity for the democratization of scientific work and a space for opportunity rather than hindrance. In recognition of the risk of the "bureaucratization" of RRI, the monitoring and evaluation system within MoRRI and the follow-up project of SUPER MoRRI seek to be as open, context-dependent, and amenable to the needs of users as possible. 


\section{Ingeborg Meijer and Wouter van de Klippe}

Nevertheless, we acknowledge that there were irreducibly political decisions made throughout the project (whom to enlist for surveys, which questions to pose, how to pose those questions, how to depict their results, which value systems to adopt in the framing of all of these actions, etc.) which concomitantly open and close potential visions of what RRI is and will become. One framing decision that was made throughout the MoRRI project was the use of the RRI framework articulated by the EC which uses six RRI keys to orient work. The next section will describe in detail the ways in which these keys were used throughout MoRRI.

\subsection{RRI keys}

The first phase of the MoRRI project required a review of the theoretical literature which underlies the six RRI keys which make up the EC's operationalization of RRI. Subsequent to this literature review, a stocktaking and assessment of potential sources of data for each key were conducted. Below, we continue with a brief discussion of each of the six keys and the theoretical work which has been developed around them.

\subsubsection{Public engagement (PE)}

The definition of the key public engagement in RRI that is featured on the EC's website is as follows: "[Public engagement] is about co-creating the future with citizens and civil society organizations, and also bringing on board the widest possible diversity of actors that would not normally interact with each other, on matters of science and technology" (EC, 2019b). The work that has investigated public engagement with science and technology is diverse, multifaceted, and often contentious.

The Royal Society published a report entitled "The public understanding of science" (1985) which is often held as a point of orientation for the history of the development of the field. Within this report, increasing the public understanding of science was advocated primarily for two reasons. First, due to the ways in which science and technology have permeated contemporary society, citizens benefit from a general understanding of science when navigating their lives. Second, a higher level of public understanding of science contributes positively to a nation's innovation system, and thus has positive implications for national prosperity.

More recent work in the field of public engagement with science and technology has articulated various problematic assumptions which were contained within the Royal Society's 1985 report. For a discussion on the developments of the field up until a decade after the report's publication, see Wynne (1995). Central to the development of the field were the identification and critique of what has been labelled the "deficit model" of public engagement with science and technology (Wynne, 2006). In short, the deficit model assumes that whatever tensions may exist within the relationship 
between the scientific community and "lay" publics is due to the "lay public" being ignorant of science, thus exemplifying a knowledge "deficit" (Bucchi, 2008). The task then, according to this model, is to remedy this tension by informing this supposedly ignorant public. What is made invisible within this model of public engagement with science and technology is the very real possibility that, when tensions exist between publics and science, this may be due to differences in underlying value commitments which are not due to any particular lack of knowledge by "lay" publics (Wynne, 2006). If this different interpretation of the cause of tension between these communities is accepted, then the role of public engagement work changes. Rather than providing platforms to inform (ostensibly) ignorant publics, instead what is needed are venues and forms of engagement which encourage the active articulation of value disputes between publics and the scientific community. In recognition of this alternative function for public engagement with science, the following categories of public engagement work were used:

- Public communication - communicating and disseminating scientific work to public audiences

- Public activism - informing decision-makers of public concerns to inform and mobilize action according to publics' needs

- Public consultation - informing decision-makers of public opinions regarding certain topics

- Public deliberation - providing venues to facilitate group deliberation policy-related scientific work

- Public participation - providing opportunities for publics to contribute to, steer, and co-construct scientific work.

\subsubsection{Science literacy and scientific education (SLSE)}

The second RRI key operationalized within MoRRI of science literacy and scientific education is intimately related with the key of public engagement, but places more emphasis on critically inspecting the various forms through which citizens are able to "comprehend and express opinions about science, as well as the ability to contribute to "doing science" (Talmon-Gros \& Teichler, 2015). The underlying theoretical grounding for this key was also informed by the theoretical work conducted for the public engagement key, most notably the shift away from a deficit model of science literacy and scientific education towards one of co-production. This resulted in three foci within this key:

1. Science education (specifically for young audiences) covering contemporary scientific work (textbook knowledge) and norms and values of science

2. Science communication work to generate awareness among citizens of all ages about science and science-related issues 


\section{6}

Ingeborg Meijer and Wouter van de Klippe

3. Science communication work with the aim of encouraging and investigating practices of co-production of scientific work with citizens.

Similar to the model used within the public engagement key, the form of science literacy and scientific education work envisioned to be monitored within MoRRI is one that enables a more inclusive R\&I environment which aims to ensure the public as an active participant and steward of science and innovation work.

\subsubsection{Gender equality (GE)}

The gender equality key within the EC's operationalization of RRI contains three objectives (EC, 2019c):

1. Ensuring that an equal gender balance is found within research teams. This is to address systemic inequalities in the makeup of research teams which have historically contained more men than women. Importantly, this objective not only emphasizes the general balance, but also attends in particular to the gender balance at different institutional and managerial levels within the R\&I environment.

2. Decision-making environments must also be more equitable in terms of gender balance. This means that, when panels, work groups, and any other decision-making space are created, these must be representative in terms of gender equality.

3. The integration of the gender dimension in R\&I content. This will extend the relevance of R\&I outputs and increase the overall quality of these outputs. Furthermore, this integration will address the systemic lack of attention paid to gender-specific concerns within the production of knowledge.

Within the MoRRI project, the functional definition of the gender equality key of RRI was theoretically extended beyond that of the EC. MoRRI understood gender through a social constructivist lens, emphasizing that it is a social construct enacted through performative practices (West \& Zimmermann, 1987; Butler, 1990). Recognizing that gender is a social construct requires attending to the ways in which gender categories are replicated in social practices. Additionally, this forces us to attend to other structural categories which are inseparably linked to gender, such as age, socio-economic status, race / ethnic background, disability, sexual preference, etc. (Harding, 2006). Consequently, MoRRI operationalized gender through an intersectional approach (Hancock, 2007).

\subsubsection{Open access $(\mathrm{OA})$}

Within the EC, the RRI key open access refers to "the global shift towards making research findings available free of charge for readers" (EC, 2019a). 
The underlying logic supporting the move to make research findings available to all, free of charge for access, is often justified through claims that this will facilitate the development of a more efficient R\&I system. This is envisioned through facilitating access to research findings for actors and institutions within the public and private sector who would, presumably, benefit from having access to these research findings. The EC put forth a recommendation to all Member States that all publicly funded research results should be made accessible to the public as early as 2012 (European Commission, 2012). The EC has since moved to an even more ambitious model of open science beyond only envisioning the findings of research to be made open to the public. This would entail, when appropriate, that both research processes and products be made more open and transparent.

Within MoRRI, the concept of open access was grounded upon the general policy concept of open science and sought to focus on the lack of available policies, frameworks, institutional tools, and institutional conditions, to encourage the opening of research processes and products (van den Eynden $\&$ Bishop, 2014). In particular, the indicators that were developed focused on the following:

- Open-access instruments for publications: these included indicators focusing on measuring publication practices within open-access journals (gold open access) and efforts for self-archiving in repositories (green open access), and thus focused predominantly on making outputs accessible;

- Developments in open-research data: These indicators used the FAIR (findable, accessible, interoperable, and reusable) framework to provide measures of the extent to which data produced within research processes were made available and usable according to ways in accordance with open-science principles. When compared to the research findings made available through open-access principles, data and datasets which have been made available and usable according to these principles are relatively scarce (Costas et al., 2013; Farhan et al., 2013).

\subsubsection{Ethics}

Within the MoRRI project's analytical report on the RRI key of ethics, the following working definition was operationalized: "Ethics as a scientific discipline is concerned with normative rules for everybody, which other than moral should be used to evaluate action and not to guide actions", which was taken from Griessler and Littig (2006, p. 134; translated by the author). However, an alternative definition supplemented this one as it was found to be more applicable within the context of R\&I: "a common platform of right and wrong, [which] is influenced by cultural norms, and aims at informing policy making" (Ladikas et al., 2015, p. 3). Note that, according to this latter definition of ethics, importance is placed on the local specificity and need for context-specific considerations when deliberating norms and notions of what is right or wrong. 
In order to draw out what normative considerations to take into account, while attending to culturally specific norms, an importance was placed on providing platforms to express ethical concerns held by different stakeholder groups. While this can be done according to formal deliberation in institutionalized settings (such as in policy-making at global, European, and national levels), this can also be done within more informal levels as well (Felt et al., 2009). This more informal engagement with ethics is often done through, for example, engaging diverse groups of individuals who may have a stake in the development of R\&I in participatory and open public engagement exercises. There must be an emphasis placed on openness, transparency and accountability of processes, explicitly considering the needs of marginalized stakeholder groups, and other considerations within these efforts.

The operationalization of ethics according to this understanding was further categorized as the following:

- Ethical governance: the institutionalization of ethics through, for example, the development and implementation of standards in R\&I policies (Brom et al., 2015), often done by ethics commissions, ethical codes, and soft law

- Ethical deliberation: the institutionalization of ethics through the facilitation of debate, among a diversity of different stakeholder groups, on issues pertaining to the development of science and technology, often taking place in advisory bodies

- Ethical reflection: the institutionalizing of ethics through providing venues for the reflection. This often takes the form of collective academic and societal reflection of ethical issues.

\subsubsection{Governance}

The MoRRI project's operational definition of governance (Stilgoe \& Lindner, 2015) emphasized various aspects of the governance of science and innovation. First, "governance" was understood as consisting of "any form of control or management", recognizing that a broader understanding of governance includes the establishing of goals, setting up means, and assessing the performance of the undertaken steps to accomplishing the established goals. The project recognized that governance exists beyond institutions of the state, emphasizing that it can and does exist at the level of individual institutions (including non-profits, universities, RFOs and RPOs, and more). Furthermore, it is emphasized that governance need not take the form of explicit rules for guidance or regulation and is also performed in other social contexts which may be more or less explicit (Gluckman \& Wilsdon, 2016).

The way that the notion of governance was understood throughout the MoRRI project also recognized that systems of governance impact each of 
the other keys which are included in RRI. As a consequence, governance in this sense should be understood as having implications on the other keys. The tools of governance which are recommended for supporting RRI are broadly in tune with a form entitled "anticipatory governance" (Barben et al., 2008, Guston, 2014) and include tools such as public deliberation methodologies, lay membership within expert committees, transparency guides, and forms of encouraging multidisciplinary collaboration.

\subsection{Development of indicators}

After these operational definitions of the EC's six keys were developed, the next task for the MoRRI project was the development of indicators which would provide insight into the ways in which these various aspects of RRI were developing across the European research area. For a more detailed discussion on the development of these indicators, please see Woolley and Rafols (2016).

Ultimately, the monitoring framework developed within the MoRRI project consisted of 36+ indicators. The indicators are listed in Table 7.1. Thirteen of the 36 indicators used readily available secondary data sources (emphasized with bold font), while 23 of the indicators required primary data collection. Many of the indicators which were developed throughout the MoRRI project required data collection at the institutional level and were subsequently aggregated at the national level. Table 7.2 provides an overview of the various methods of primary data collection which were used in the MoRRI project; these methods ranged from surveys of RPOs, RFOs, and societal stakeholders; bibliographic data and - to a lesser degree - data based on qualitative approaches (case studies). The secondary data set applied and collected in the MoRRI project include data from Eurostat, SheFigures, Eurobarometers, OpenAIRE, and specific cross-European projects such as MORE2, MASIS, EPOCH, and SATORI.

An important note should be made regarding the fact that there exists considerable overlap between the different RRI keys. The MoRRI project undertook the assumption that, while these keys can be distinguished as a useful heuristic for the purposes of implementation, they are intimately related and not independent of one another. As a consequence of the overlap between the keys, there are likely relationships between the different indicators which were developed to measure the different keys. An illustration of the potential links and overlaps between the different keys and the indicators developed to measure them is provided in Figure 7.1. The arrows with full lines within Figure 7.1 indicate that these links were directly assessed in the development of the indicators, whereas the arrows with dashed lines indicate links which require additional theoretical exploration. The direction of the arrows indicates the hypothesized direction of influence between the links, and also shows whether or not there is theorized uni-directionality or bi-directionality in the relationship between the indicators / keys. 
Table 7.1 Indicators capturing aspects of Responsible Research and Innovation (RRI)

\begin{tabular}{|c|c|c|}
\hline Key & Indicator & Indicator title \\
\hline \multirow{10}{*}{$\begin{array}{l}\text { Gender } \\
\text { equality }\end{array}$} & GE1 & Share of RPOs with gender equality plans \\
\hline & GE2 & $\begin{array}{l}\text { Share of female researchers by sector } \\
\text { (secondary data) - four sub-dimensions }\end{array}$ \\
\hline & GE3 & Share of RFOs promoting gender content in research \\
\hline & GE4 & $\begin{array}{l}\text { Dissimilarity index (secondary data) - two } \\
\text { sub-dimensions }\end{array}$ \\
\hline & GE5 & $\begin{array}{l}\text { Share of RPOs with policies to promote gender in } \\
\text { research content }\end{array}$ \\
\hline & GE6 & Glass ceiling index (secondary data) \\
\hline & GE7 & Gender pay gap (secondary data) two sub-dimensions \\
\hline & GE8 & Share of female heads of RPOs \\
\hline & GE9 & Share of gender-balanced recruitment committees at RPOs \\
\hline & GE10 & $\begin{array}{l}\text { Number and share of female inventors and authors - two } \\
\text { sub-dimensions }\end{array}$ \\
\hline \multirow{4}{*}{$\begin{array}{l}\text { Science } \\
\text { literacy and } \\
\text { education }\end{array}$} & SLSE1 & $\begin{array}{l}\text { Importance of societal aspects of science in science } \\
\text { curricula for } 15-18 \text {-year-old students (desk research) }\end{array}$ \\
\hline & SLSE2 & RRI-related training at higher education institutions \\
\hline & SLSE3 & Science communication culture (secondary data) \\
\hline & SLSE4 & Citizen science activities in RPOs - two sub-dimensions \\
\hline \multirow[t]{3}{*}{ Ethics } & & Ethics at the level of RPOs - two sub-dimensions \\
\hline & E2 & National ethics committees index (secondary data) \\
\hline & E3 & RFO ethics index - two sub-dimensions \\
\hline \multirow[t]{10}{*}{$\begin{array}{l}\text { Public } \\
\text { engagement }\end{array}$} & PE1 & $\begin{array}{l}\text { Models of public involvement in science and technology } \\
\text { decision making (secondary data) }\end{array}$ \\
\hline & PE2 & Policy-oriented engagement with science (secondary data) \\
\hline & PE3 & $\begin{array}{l}\text { Citizen preferences for active participation in science and } \\
\text { technology decision making (secondary data) }\end{array}$ \\
\hline & PE4 & $\begin{array}{l}\text { Active information search about controversial technology } \\
\text { (secondary data) }\end{array}$ \\
\hline & PE5 & $\begin{array}{l}\text { Public engagement performance mechanisms at the level } \\
\text { of research institutions }\end{array}$ \\
\hline & PE6 & $\begin{array}{l}\text { Dedicated resources for public engagement - indicator } \\
\text { dropped }\end{array}$ \\
\hline & PE7 & $\begin{array}{l}\text { Embedment of public engagement activities in the funding } \\
\text { structure of key public research-funding agencies }\end{array}$ \\
\hline & PE8 & $\begin{array}{l}\text { Public engagement elements as evaluative criteria in } \\
\text { research proposal evaluations }\end{array}$ \\
\hline & PE9 & Reseach and innovation democratization index \\
\hline & PE10 & $\begin{array}{l}\text { National infrastructure for involvement of citizens and } \\
\text { societal actors in research and innovation }\end{array}$ \\
\hline \multirow[t]{6}{*}{ Open access } & OA1 & Open access literature - two sub-dimensions \\
\hline & OA2 & Data publications and citations - indicator dropped \\
\hline & OA3 & $\begin{array}{l}\text { Social media outreach /take-up of open access literature - } \\
\text { two sub-dimensions }\end{array}$ \\
\hline & OA4 & Public perception of open access (secondary data) \\
\hline & OA5 & Funder mandates (secondary data) \\
\hline & OA6 & $\begin{array}{l}\text { RPO support structures for researchers as regards } \\
\text { incentives and barriers for data sharing }\end{array}$ \\
\hline \multirow[t]{3}{*}{ Governance } & GOV1 & $\begin{array}{l}\text { Composite indicator: governance for responsible research } \\
\text { and innovation (secondary data) }\end{array}$ \\
\hline & GOV2 & $\begin{array}{l}\text { Existence of formal governance structures for RRI within } \\
\text { RFOs and RPOs }\end{array}$ \\
\hline & GOV3 & Share of RFOs and RPOs promoting RRI \\
\hline
\end{tabular}

Notes: RFOs, research-funding organizations; RPOs, research-performing organizations. 
Table 7.2 Overview of primary data collection methods

\begin{tabular}{ll}
\hline Methods for collecting primary data & Indicators \\
\hline Science in Society actor survey & PE9, PE10 \\
$\begin{array}{l}\text { Higher education institutions and } \\
\text { research-performing organizations survey }\end{array}$ & GE1, GE5, GE8, GE9, SLSE2, SLSE 4, \\
$\begin{array}{l}\text { Research-funding organizations survey } \\
\text { Register-based data }\end{array}$ & GE3, PE7, PE8, E3, GOV2, GOV3 \\
$\begin{array}{l}\text { Qualitative data, interviews/desk research } \\
\text { GE10, OA1, OA2, OA3 }\end{array}$ & SLSE1 \\
\hline
\end{tabular}

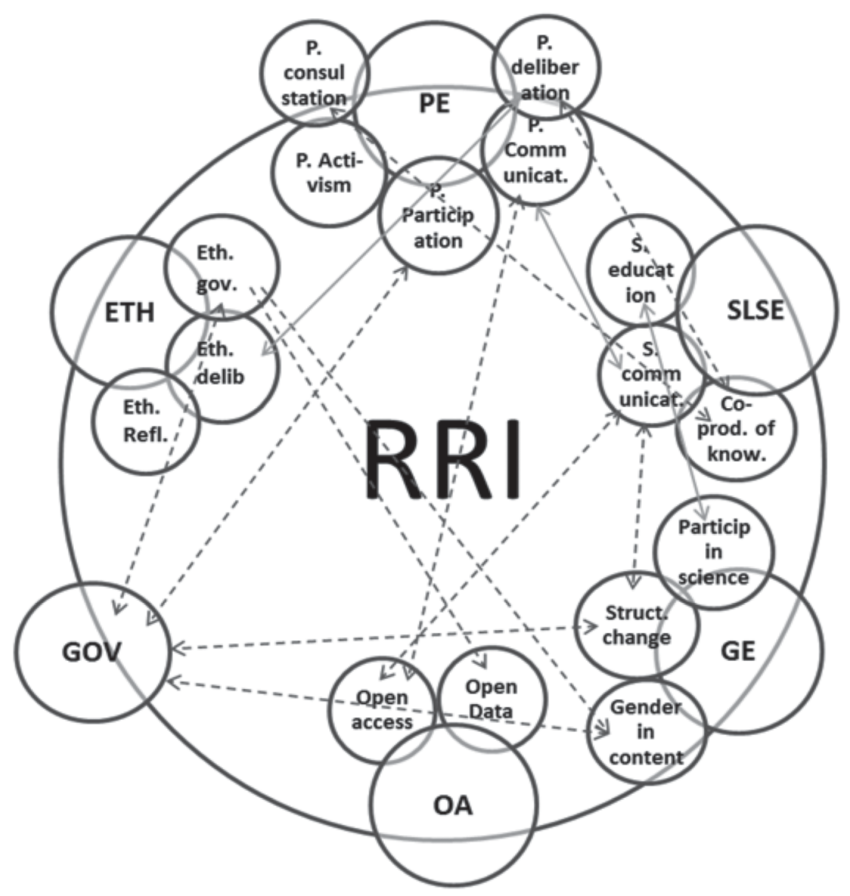

Figure 7.1 Existing and potential interlinkages/overlaps between Responsible Research and Innovation (RRI) (sub-)categories (Taken from Meijer et al., 2016 and Peter et al., 2018.)

\subsection{Emerging patterns at the country level}

"The Evolution of Responsible Research and Innovation in Europe: The MoRRI Indicators Report" (MoRRI Consortium, 2018; Peter et al., 2018) presents the results of individual indicators and the monitoring of developments. The methodological background to that analysis is described in more detail in Mejlgaard et al. (2019). The data collection aimed to populate 


\section{Ingeborg Meijer and Wouter van de Klippe}

the 36+ indicators covering the six keys of RRI. Subsequently, the empirical structure of the data collected was analysed with the purpose of understanding the appropriateness of the indicators towards the "key" dimensions that they were intended to be indicators of. For the year 2016 a cross-sectional dataset was available covering all $28 \mathrm{EU}$ countries. A validation procedure was conducted to test four main aspects: the quality of indicators based on surveyquestions (removal of indicators based on items with high non-response); the internal consistency of composite measures; the robustness of indicators on the basis of testing the effects of small adjustments in indicator specifications on ranking; and the degree of within-country variance compared to crosscountry variance. While the coverage of the indicators across EU countries is comprehensive, the majority of indicators have a few missing cases, i.e. countries for which no observed value has been obtained. Also, on country level, there are more indicators than observations, which presents a challenge to any integrated statistical models drawing on multiple indicators. For the examination of the empirical patterns of the indicators, these limitations have been accommodated by imputing data points to replace missing values, and applying factor analyses to subsets of indicators rather than the full set of 36+ indicators. Principal component factor analyses were conducted for each key area separately. The aim of the imputation was to examine how the selected indicators relate to each other and to the dimensions one would expect them to be indicators of, in order to address integrated empirical findings.

The purpose of the factor analysis was to determine the number of distinct dimensions for each key. A second aim was identification of the indicators that are most relevant in capturing these dimensions. Interestingly, the parallel principal component analyses revealed two distinct and interpretable factors for all keys except governance, for which only one factor was retained. In Table 7.3, the 11 retained sub-dimensions are presented, along with the indicators most relevant to the respective sub-dimension.

Table 7.3 Responsible Research and Innovation (RRI) keys and core indicators

\begin{tabular}{ll}
\hline Dimension & Core indicators \\
\hline GE action & GE1, GE5 \\
GE status & GE2.3, GE10.1 \\
SLSE training & SLSE1, SLSE2 \\
SLSE culture & SLSE3, SLSE4 \\
PE participation & PE1, PE4, PE9 \\
PE in assessment & PE7, PE8 \\
Ethics in RPOs & E1a, E1b \\
Ethics in RFOs & E3a, E3b \\
OA status & OA1.1, OA1.2 \\
OA action & OA3, OA4, OA6 \\
Governance & GOV1, GOV2, GOV3 \\
\hline
\end{tabular}

Source: Peter et al. (2018). 
Interestingly, the two dimensions for each key represent two aspects. One aspect is related to policies, action, activities, or planning to support or enhance an RRI key. The other one relates to the actual status, situation, or culture of the RRI keys. For example, for the key gender equality, the first dimension that materialized from the principal component analyses was labelled GE actions. This combines GE1, which measures the share of RPOs with gender equality plans, and GE5, measuring the share of RPOs with policies promoting gender content in research. The second sub-dimension of gender equality represents the actual GE status and is strongly interrelated with GE2.3 and GE10.1. They measure the share of female researchers in the higher education sector and the share of female authors of scientific papers, respectively. GE status is not about policies to promote gender equality but rather about the status observed concerning female representation in science.

Science literacy and science education was also divided into two dimensions. One relates to formal training activities around issues of responsibility in secondary education (SLSE1) and in higher education institutions (SLSE2) and can be called SLSE training, representing the "action". The other - the actual situation - is about the broader national science culture, indicated by SLSE3 capturing aspects of science communication culture and SLSE4 signalling the importance of citizen science activities in RPOs (thus, SLSE culture).

The "action" in public engagement revolves around citizens' active participation. Three indicators of public involvement in science and technology decision-making (PE1), citizens' search for information about controversial technologies (PE4), and the level of democratization of R\&I (PE9) all pertain strongly to this sub-dimension. The "situation" sub-dimension is mainly concerned with the extent to which public engagement is included in assessment exercises. This dimension relates strongly with PE7, which measures the inclusion of public engagement activities in the activities of RFOs, and PE8, which is about the extent to which public engagement is used as evaluation criteria in the assessment of research proposals.

For the ethics key the first sub-dimension is concerned with planning, i.e. the existence of (E1a) and degree of importance attributed to (E1b) research ethics committees and research integrity offices at higher education institutions and other public RPOs (ethics in RPO). The second dimension (ethics in RFO) is more concerned with the culture around ethics in RFOs. It is informed by two indicators relating to the use of an ethics review in relation to funding decisions (E3a) and a composite indicator based on a set of questions relating to the importance of such assessment for funding decisions (E3b).

Almost equivalent to the pattern from gender equality, open-access indicators seem to support the existence of two dimensions capturing "status" and "action". There is a sub-dimension concerning activities promoting open access (actions), such as OA3 on social media outreach or 


\section{Ingeborg Meijer and Wouter van de Klippe}

uptake of open-access literature, OA4 on public support for open access to scientific information, and OA6 on support structures promoting data sharing within RPOs. They all relate to "open-access activities" which could be expected to push a Member State towards higher levels of open access. The OA sub-dimension concerning the state of play consists of indicators OA1.1 and OA1.2 measuring the share of open-access publications as proportions of all publications in a country.

Finally, the three indicators in the governance key end up in one single factor. GOV1 concerns the use of science in policymaking at the national level, while GOV2 addresses RRI-related formal governance mechanisms within RPOs and RFOs. GOV3 is a composite measure of institutional support for RRI among employees within these organizations. The empirical structure of the governance indicators thus supports retaining only one RRI governance dimension, which is in line with the actual interlinkage of governance with all other keys.

Thus, from the $36+$ original indicators, 25 proved to be strong indicators for the11 RRI sub-dimensions that emerged empirically from the data. For those 25 indicators, a $0-1$ normalized index was subsequently created, which was then used to characterize individual countries for the 11 sub-dimensions. In turn, the 11 indexes helped to explore similarities and differences between and within clusters of countries. The approach to cluster analysis of countries was a hierarchical, weighted average linkage cluster analysis.

Figure 7.2 presents the clusters, based on country scores on the 11 indices. The dendrogram reveals a somewhat heterogeneous set of countries;

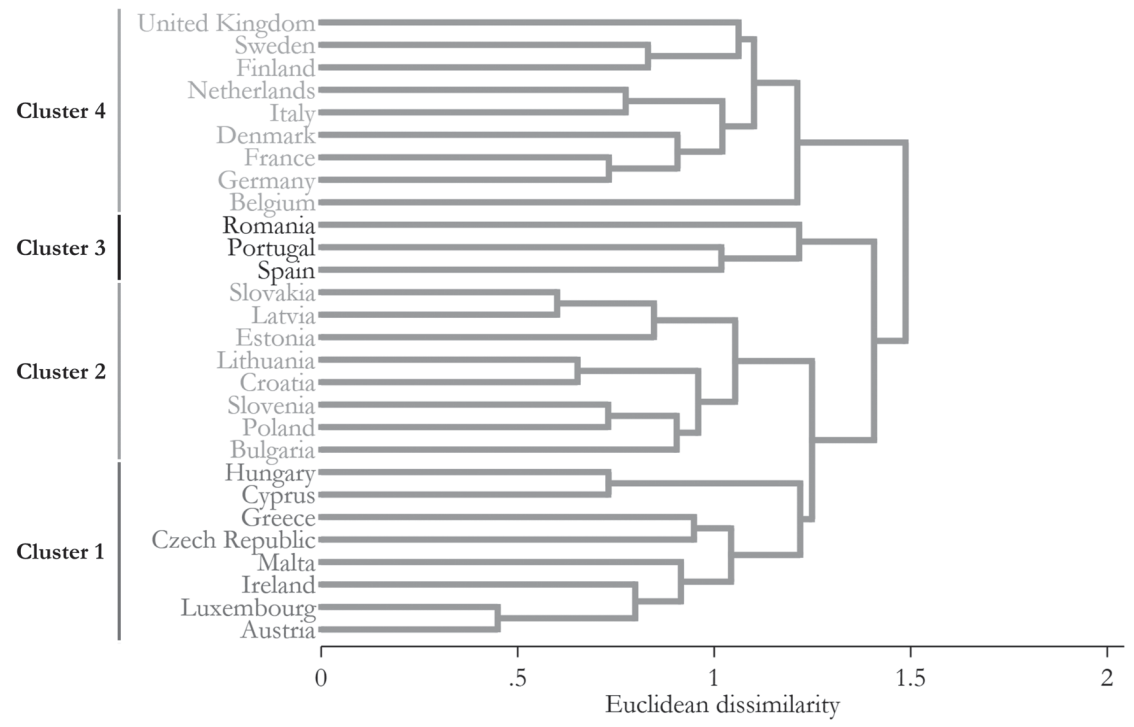

Figure 7.2 Dendrogram of country clustering. (Taken from Peter et al., 2018.) 
looking horizontally from left to right, each Member State initially forms its own cluster but progressively Member States cluster together based on the average linkage algorithm, resulting in continuously fewer clusters. The four-cluster solution seems most useful from an interpretation perspective. Looking from the bottom up, Austria, Luxembourg, Ireland, Malta, the Czech Republic, Greece, Cyprus, and Hungary form a first cluster. This means that their individual country profiles are alike, and that they as a group are distinct from the other groups. The second group includes Bulgaria, Poland, Slovenia, Croatia, Lithuania, Estonia, Latvia, and Slovakia. The third and smallest group includes Spain, Portugal, and Romania. The fourth and last group includes Belgium, Germany, France, Denmark, Italy, the Netherlands, Finland, Sweden, and the United Kingdom.

In Figure 7.3, the characteristics of the four clusters are displayed as a radar plot showing the profile of the 11 RRI dimensions for each cluster of Member States. For each of the 11 sub-dimensions it captures the range from minimum to maximum possible effort within the sub-dimensions. The use of a clustering algorithm to group countries does not mean that countries within a cluster have exactly the same RRI profiles. Rather, it means that the profile of a country within a cluster is more alike to other members of that cluster than to countries belonging to a different cluster. However,

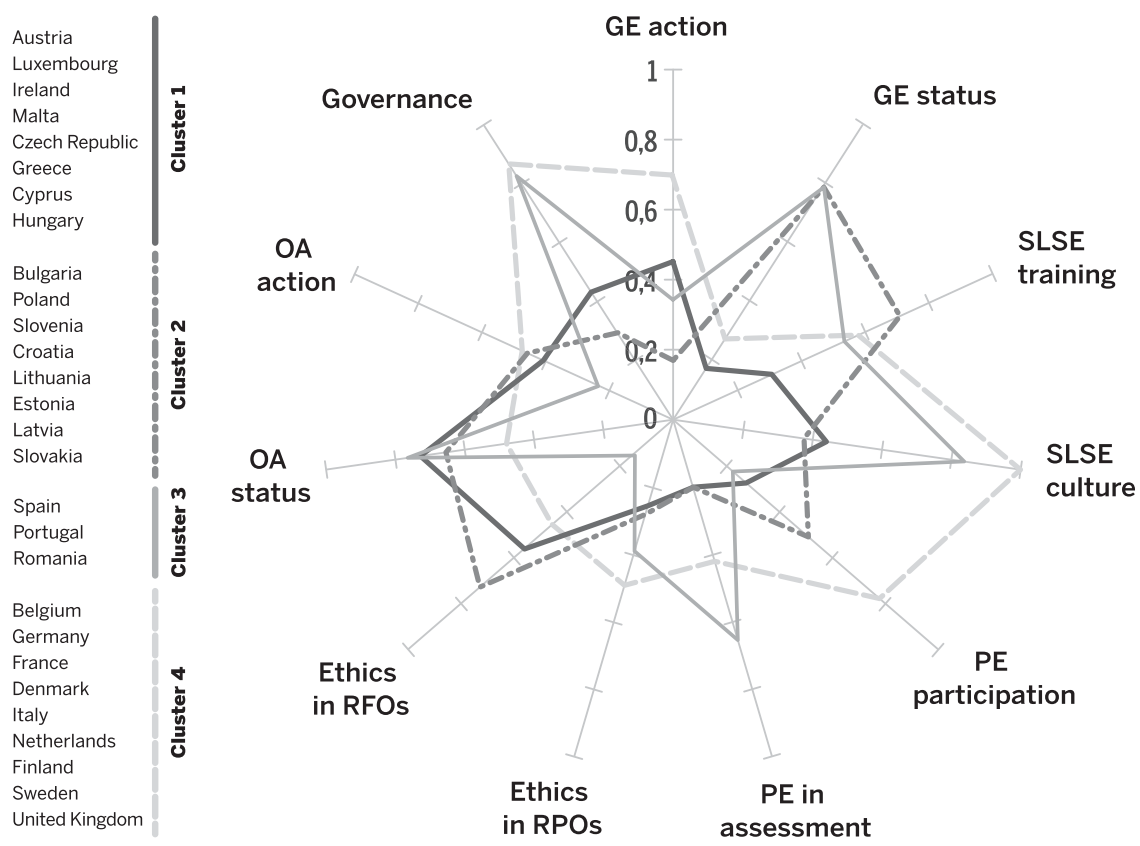

Figure 7.3 Responsible Research and Innovation (RRI) characteristics of four clusters of EU Member States. (Taken from Peter et al., 2018.) 
there can be significant differences in the country profiles, even within the same cluster.

The first cluster of Member States is characterized by having belowaverage scores on most of the 11 RRI dimensions apart from "OA status" and "ethics in RFOs", where this cluster is performing relatively well. Within this cluster, there is a rather moderate level of accomplishment concerning RRI in general.

The second cluster of Member States performs particularly well on "GE status", "SLSE training", and "ethics in RFOs", and also rather well on both sub-dimensions of open access. At the same time, the average score of this cluster on "GE action" and "governance" is considerably lower than for the other clusters.

The third cluster resembles the shape of a star due to its high scores on nearly every second dimension and fairly low scores on the other half. On one dimension, "PE in assessment", this cluster is doing particularly well. Additionally, Member States within this cluster tend to score very highly on both gender equality status and open-access status.

The fourth cluster is generally performing above average. Exceptions include the dimensions of "GE status" and "OA status" where the average score of Member States within this cluster is low. Sub-dimensions related to inclusivity and co-creation of R\&I with civil society (PE participation and SLSE culture) perform particularly well.

Interestingly, for both clusters 2 and 3 , there is a negative relation between gender equality status and action. Countries with a high level of observed gender equality in science (according to our indicators) are seemingly less prone to be highly active regarding gender equality policies and action plans at the institutional level. In countries in which the historical labour market trajectories have been more conducive to gender equality in science, there may be less need for action. However, these conclusions are tentative and these results must be examined with the broader context of the history of gender equality in science.

Remarkably, three clusters (1, 2, and 3 ) have similarly high scores for OA status, which indicates that the use of open access for scientific publication relative to all publications is high across these clusters. This is however not the case in cluster 4, where open-science policies are prominent, such as the UK, the Netherlands, and Finland, but where the use of open access for publications is lower than in the other three.

On the one hand, the results of the cluster analyses show that there is significant diversity in the European RRI landscape, with unequal distribution across the 11 sub-dimensions and across Member States. Clearly, the origins of such diversity cannot be read from the graphics, but require further investigation of historical trajectories in the relationship between science and society, R\&I policy approaches, as well as political and civic culture. Some distinctions, e.g. between the primarily north-western European cluster 4 and the primarily eastern European cluster 2, seem to be in line with earlier findings concerning science's role and responsibilities in society. 
On the other hand, and despite the careful imputation of missing data, findings may not capture the diversity between the responding institutes across and within Member States. Research institutes have other priorities than higher education institutes with a significant teaching load; large general and traditional universities with a broad spectrum of research fields have different needs than specialized or technical universities; and the location of an institute in an urban or rural setting may induce various local or regional contexts. Especially when the number of observations does not exceed the number of indicators, a country profile may be the result of the (local) context of the responding institutes. If a realistic perspective of RRI is the aim, it makes sense to take the institutional level more centrally in further monitoring of RRI. Eventually, the institutional context is leading in responding to and implementation of policies that support the anticipation, reflection, responsiveness, and inclusivity of R\&I.

\subsection{Exploring the benefits of RRI}

The MoRRI project also sought to investigate benefits which could be attributable, to some extent, to the implementation of changes in line with the aims of RRI. For a more detailed discussion on these potential benefits, see Wuketich et al. (2016).

Before describing the benefits which were observed, a theoretical and methodological note should be made. While the indicators which were developed throughout the MoRRI project were developed with a relatively conventional intervention logic (inputs, outputs, outcomes, and impacts), it was recognized that conceptualizing the benefits of RRI in this same logic would likely result in missing some of the eventual effects of its implementation.

The recognition of the fact that benefits cannot be immediately envisioned within this inputs-to-impacts intervention logic led to two elements which were of particular note when trying to attend to the benefits of RRI. The first element of the benefits of RRI which was recognized was the observation that frequently RRI benefits manifest themselves in the transformations of existing processes. For example, if RRI-informed policy-making resulted in the institutionalizing of a public engagement platform within an RPO, with the explicit desire to empower previously disenfranchised populations within research processes, this can be understood as a democratic benefit of RRI. The novel space for the enfranchisement of these actors within this institutionalized engagement exercise can be thought of as a potential RRI benefit irrespective of other additional substantive outcomes. As this example highlights, when one seeks to investigate the potential benefits of RRI, it is crucial to attend to forms of institutional change.

A second element which requires extending the interpretation of the benefits of RRI beyond an input impact model is that the benefits of RRI should be understood as being normative in character. Indeed, the question of how to align R\&I with the needs of society is context-dependent, and requires asking detailed questions such as: Which actors in society do 
we mean? Whose needs are excluded? among other irreducibly political questions. Therefore, observing the benefits of RRI cannot be understood through a framework of being universally "positive", "net positives", or by measuring accumulated effects (Peter et al., 2018). Consequently, the MoRRI project used detailed case studies to identify potential benefits of RRI, and focused on understanding institutional change and attending to the different actors for whom these changes benefitted.

Throughout the case studies, the identified benefits were categorized according to four potential types. This typology consisted of societal benefits, democratic benefits, economic benefits, and scientific benefits. Although these four different categories of RRI benefits were identified, these are to be understood as a useful typology rather than a prescriptive set of benefit categories; the benefits of RRI are unlikely to be neatly distributed among these distinct categories and will likely transcend or evade the categories of this typology. For a detailed description of the case studies that were completed throughout the MoRRI project, please see Wuketich et al. (2016).

A principal democratic benefit that was identified in the case studies was the increased participation of a diversity of actors within the R\&I system. By institutionalizing public engagement exercises within R\&I processes, actors who otherwise would not have been able to express their values and contribute to the steering of R\&I were able to do so. One case study exemplifying the institutionalization of public engagement was a large research project entitled "Building Adaptive Strategies for Environmental Change with Rural Land Managers" which adopted a participatory action research approach (Pain, Whitman, \& Milledge, 2011). This project enlisted land managers from the outset and ensured that they played an active role in shaping the research project and contributed to knowledge production and tool development which would subsequently be used within policy-making contexts. In this example, the production of knowledge itself was done through a more democratic and inclusive way, highlighting the democratic benefits for the participating land managers.

Of the societal benefits which were identified throughout the MoRRI project, one of the most evident was the inclusion of gender-specific concerns within research processes. One of the case studies conducted throughout MoRRI involved researching the Institute of Gender in Medicine at the Charité University of Medicine in Berlin. The head of the institute led efforts to actively address the fact that women experience significantly worse outcomes regarding the diagnosis and treatment of cardiovascular diseases (Regitz-Zagrosek, 2012). One of the outcomes of this initiative was the creation of novel courses to train clinicians to identify gender-specific concerns and address inequalities in the ways in which diagnosis and treatment were performed. Additionally, research projects were developed which explicitly sought to investigate the different ways in which cardiovascular diseases manifest depending on gender.

With regards to the economic benefits of RRI, these were particularly difficult to measure. However, the MoRRI project investigated the growth of 
open-access publications within Denmark, the Netherlands, and Switzerland between the years of 2000 and 2012. It was found that the proportion of publications which were published open-access increased in each country during this time period. Although the direct economic benefits of this change are difficult to assess, it can be assumed that, when these publications were utilized by firms, there was an economic benefit for firms when they no longer had to pay access fees. Lowering the barrier to being able to access these publications may facilitate the transfer of knowledge between the academic and industrial sectors, having eventual economic benefits.

Finally, scientific benefits were observed in a number of case studies throughout MoRRI. The term "scientific benefits" is vague, and a rather open-ended operationalization of the term was used throughout the description of the case studies. With regard to scientific benefits observed through the implementation of efforts to consider gender-specific concerns in research, it was noted that the knowledge produced because of these institutionalized changes was beneficial in that it addressed concerns and gaps which otherwise would have been ignored. Additionally, the knowledge which was coproduced with diverse populations through the institutionalization of public engagement exercises benefitted from the diverse forms of expertise which were able to voice their needs within the R\&I system. One example of the scientific benefits of the institutionalizing of public engagement exercises was evident in the analysis of the GAP2 project. The GAP2 project enlisted fishers within a research project investigating sustainability issues in fisheries. As a consequence of the inclusion of these actors within the research process, the participating researchers quickly found that it was not a process of informing fishers of novel developments within research, but instead that there was a benefit in efforts to "engage fishers as active agents in the knowledge production process". Evidently, the forms of knowledge and expertise which the fishers held would not have contributed to the production of scientific knowledge if these public engagement opportunities had not been created (see Wuketich et al., 2016).

As mentioned above, the separation of potential benefits that the implementation of RRI had into these categories is merely a useful typology. The benefits which occur due to RRI are likely to be intimately linked and extend well beyond the discussion above. The interlinked nature of these benefits, and several benefits which were not discussed, can be observed in Figure 7.4.

\subsection{The future of monitoring RRI}

RRI is not the first (policy) concept with the lofty aims of creating a more socially responsive R\&I system and one can reasonably state that its implementation has been unsuccessful in comprehensively changing R\&I practices. Indeed, hearing innovation myths which are antithetical to the underlying philosophy of RRI, such as R\&I being politically neutral, or the continued use of linear models of innovation is still commonplace (for a rich discussion 


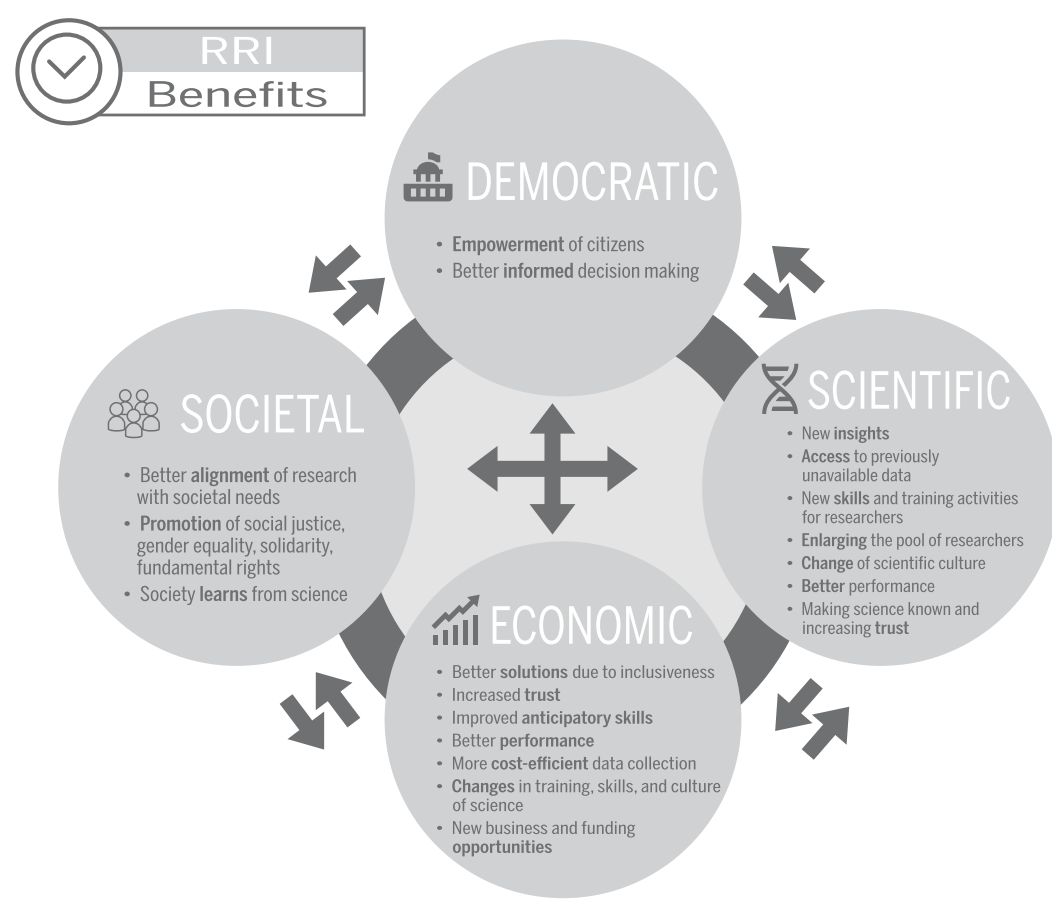

Figure 7.4 Societal, democratic, economic and scientific benefits of Responsible Research and Innovation (RRI). (Taken from Peter et al., 2018.)

of other oft-repeated innovation myths, see Joly, 2019). Even more problematic is the concern that RRI risks serving as "responsible washing" because of its voluntary and highly flexible character.

Nevertheless, the concept of RRI has considerable momentum and political weight behind it, and is at present a prominent device employed throughout a diversity of international research efforts, notably within the context of EC funding schemes (Smallman et al, 2015; EC, 2018). The growth of RRI within the R\&I system is evinced by the many projects funded through the Science with and for Society (SwafS) programme of Horizon2020, many placing key tenets of RRI, such as co-creation environments, central (Mejlgaard et al., 2018). Furthermore, a wealth of RRI literature, much of it within the Journal of Responsible Innovation, and tools guiding RRI change (such as those present on the www.rri-tools.eu web page) further highlights the vast work which is being done under the heading of RRI (see also Von Schomberg \& Hankins, 2019). It is crucial to recognize, however, that the swathe of efforts which are informed by RRI needs adjustment considering the shortcomings which have been articulated.

The question of how to continue working in the space of RRI, while acknowledging its many shortcomings, makes evident the need for continued 
reflection and negotiation of the concept of RRI itself, which has direct consequences on what a monitoring system for RRI should resemble. This question, and its multiplicity of deeply normative, political, and contextdependent answers are focal within the successor project of MoRRI: SUPER MoRRI. While the primary output of MoRRI was the creation of a monitoring system highlighting changes at the national level, the SUPER MoRRI project envisions an alternative future for the monitoring and evaluation of RRI which is much more intimately local and context-dependent.

It was through recognizing the importance of changes at the institutional level that SUPER MoRRI has shifted emphasis towards providing a monitoring framework which aims to provide knowledge and monitor changes at the institutional level rather than monitoring the state of the implementation at the national level and benchmarking nations, or clusters of nations, against each other. The case studies conducted within MoRRI highlighted that the implementation of RRI can often best be understood within an institutional context. Indeed, shifting towards a monitoring system seeking to aid in institutional change may have the benefit of empowering individuals to articulate their own values within their institutions by providing them with intellectual resources to do so. This is a considerably different view of the function of a monitoring framework - one that shifts away from simply measuring and actively moves towards intervening and changing practices.

RRI takes as an assumption that fact that science, technology, and society mutually co-construct each other. Against the backdrop of entrenched social inequalities, environmental degradation, and socio-political unrest, the R\&I system has a duty to become more responsive to societal needs. Despite the limitations of RRI, its political weight is considerable, and the opportunity that it has to reconstruct an R\&I system which is more just is yet to be determined. RRI may indeed be a venue for opening up a space within the R\&I system for asking deeply normative questions and allowing for the enfranchisement of voices to elicit their needs within R\&I. The task of the SUPER MoRRI project is to develop a monitoring system to better understand how and whether this space is indeed being opened.

\section{Acknowledgement}

This chapter builds upon and summarizes work in the Monitoring the Evolution and Benefits of Responsible Research and Innovation (MoRRI) project 2014-2018 that was funded by the European Commission, contract number RTD-B6-PP-00964-2013, duration 09/2013-03/2018. We are greatly indebted to our project partners, who are equally responsible for the work carried out and described in more detail in the MoRRI deliverables. The partners are: Viola Peter, Frederic Maier (editors) (Technopolis Group); Niels Mejlgaard, Carter Bloch, Emil B. Madsen (Aarhus University); Erich Griessler, Milena Wutekich (IHS); Ingeborg Meijer (CWTS, Leiden University); Richard Woolley (Ingenio CSIC-UPV); Ralf Lindner, Susanne Bührer, Angela 


\section{Ingeborg Meijer and Wouter van de Klippe}

Jäger (Fraunhofer ISI); Lena Tsipouri (University of Athens); Jack Stilgoe (UCL). Figures in this chapter are directly taken from MoRRI deliverables with permission of the EC. Figures 7.1-7.4 and Table 7.3 appeared in the EC report: Monitoring the evolution and benefits of responsible research and innovation in Europe; Summarising insights from the MoRRI project, May 2018 (Peter et al., 2018); Tables 7.1 and 7.2 are included in the conference paper for the OECD Blue Sky 2016 paper (Meijer et al., 2016).

\section{References}

Barben, D., Fisher, E., Selin, C., \& Guston, D. H. (2008). Anticipatory governance of nanotechnology: Foresight, engagement, and integration. The Handbook of Science and Technology Studies. London: MIT Press, p. 979.

Brom, F. W. A., Chaturvedi, S., Ladikas, M., \& Zhang, W. (2015). Institutionalizing ethical debates in science, technology, and innovation policy: A comparison of Europe, India and China. In: Ladikas, M., Chaturvedi, S., Zhao, Y., \& Stemerding, D. (eds.), Science and Technology Governance and Ethics. Cham: Springer, pp. 923. https://doi.org/10.1007/978-3-319-14693-5_2

Bucchi, M. (2008). Of deficits, deviations and dialogues: Theories of public communication of science. In: Bucchi, M. \& Trench, B., Handbook of Public Communication of Science and Technology. New York: Routledge, pp. 71-90.

Butler, J. (1990). Gender Trouble. Feminism and the Subversion of Identity. New York: Routledge.

Costas, R., Meijer, I., Zahedi, Z., \& Wouters, P. (2013). The value of research data: Metrics for datasets from a cultural and technical point of view. A Knowledge Exchange Report (pp. 1-48). Available at: www.knowledge-exchange.info/event/ value-reseach-data-metrics

European Commission (EC). (2012). Commission Recommendation on Access to and Preservation of Scientific Information. Available at: http://ec.europa.eu/ research/science- society/document_library/pdf_06/recommendation-access-andpreservation-scientific-information_en.pdf

European Commission (EC). (2018). Responsible Research and Innovation. Available at: https:/ec.europa.eu/programmes/horizon2020/en/h2020-section/ responsible-research-innovation

European Commission (EC). (2019a). Open Science (Open Access). Available at: https://ec.europa.eu/programmes/horizon2020/node/1031

European Commission (EC). (2019b). Public Engagement in Responsible Research and Innovation. Available at: https://ec.europa.eu/programmes/horizon2020/node/766.

European Commission (EC). (2019c). Promoting Gender Equality in Research and Innovation. Available at: https://ec.europa.eu/programmes/horizon2020/node/797

Farhan, H., Alonso, J., Davies, T., Tennison, J., Heath, T., \& Berners-lee, T. (2013). Open Data Barometer 2013 Report, 1-45. Available at: http://opendatabarometer. org/doc/1stEdition/Open-Data-Barometer-2013-Global-Report.pdf

Felt, U., Fochler, M., Müller, A., \& Strassnig, M. (2009): Unruly ethics: on the difficulties of a bottom-up approach to ethics in the field of genomics. Public Understanding of Science, 18(3), 354-371.

Gluckman, P., \& Wilsdon, J. (2016). From paradox to principles: Where next for scientific advice to governments? Palgrave Community, 2, 16077. 
Griessler, E. and Littig, B. (2006). Neosokratisch Dialoge zu ethische Fragen der Xenotranspantation. Ein Beitrag zur Bearbeitung ethische Probleme in partizipatiever Technikfolgenabschützung. In: E. Buchinger \& U. Felt (eds.), Technik und Wissenschaftssoziologie in Österreich. Stand und Perspektiven. Österreichische Zeitschrift für Soziologie, Sonderheft. 8: S.131-157.

Guston, D. H. (2014). Understanding 'anticipatory governance'. Social Studies of Science, 44(2), 218-242.

Hancock, A. (2007). Intersectionality as a normative and empirical paradigm. Gender and Politics, 3(2), 248-254.

Harding, S. (2006). Science and Social Inequality: Feminist and Postcolonial Issues. Urbana, IL: University of Illinois Press.

Heras, M., \& Ruiz-Mallén, I. (2017). Responsible research and innovation indicators for science education assessment: How to measure the impact? International Journal of Science Education, 39(18), 2482-2507.

Italian Presidency of the Council of the European Union. (2014). Rome Declaration on Responsible Research and Innovation in Europe. Brussels, Belgium: European Commission. https://ec.europa.eu/research/swafs/pdf/rome_declaration_RRI_ final_21_November.pdf.

Joly, P-B. (2019). Reimagining innovation. In: S. Lechevalier (ed.), Innovation Beyond Technology. Science for Society and Interdisciplinary Approaches. doi. org/10.1007/978-981-13-9053-1_2

Ladikas, M., Chaturvedi, S., Zhao, Y., \& Stemerding, D. (2015). Introduction: Embedding ethics in science and technology policy - a global perspective. In: M. Ladikas, S. Chaturvedi, Y. Zhao, \& D. Stemerding (eds.), Science and Technology Governance and Ethics. Cham: Springer. doi.org/10.1007/978-3-31914693-5_1

Meijer, I., Mejlgaard, N., Lindner, R., Woolley, R., Rafols, I., Griesler, E., Wroblewski, A., Bührer, S., Stilgoe, J., Tsipouri, L, Maroulis, N., \& Peter, V. (2016). Monitoring the evolution and benefits of Responsible Research and Innovation (MoRRI) a preliminary framework for RRI dimensions and indicators. OECD Blue Sky Forum 2016. www.oecd.org/sti/105\%20-\%20Meijer_MoRRI_paper_\%20 OECD_bluesky_16ul16_def.pdf

Mejlgaard, N., Bloch, C., \& Bargmann Madsen, E. (2019). Responsible research and innovation in Europe: A cross-country comparative analysis. Science and Public Policy, 46, 198-209. DOI: 10.1093/scipol/scy048

Mejlgaard, N., Woolley, R., Bloch, C., Bührer, S., Griessler, E., Jäger, A., Lindner, R., Bargmann Madsen, E., Maier, F., Meijer, I., Peter, V., Stilgoe, J., \& Wuketich, M. (2018). Europe's plans for responsible science. Science, 361(6404), 761-762. DOI: $10.1126 /$ science.aav0400

Monsonís-Payá, I., García-Melón, M., \& Lozano, J. F. (2017). Indicators for Responsible Research and Innovation: A methodological proposal for contextbased weighting. Sustainability, 9(12), 2168.

MoRRI Consortium. (2015). Synthesis Report on Existing Indicators Across RRI Dimensions, Task 3, Progress Report, Deliverable 3.2. Available at: http://morriproject.eu/reports/2015-05-01-d3.1

MoRRI Consortium. (2018). The Evolution of Responsible Research and Innovation in Europe: The MoRRI Indicators Report. Monitoring Report (D4.3). Available at: http://morri-project.eu/reports/2018-02-21-the-evolution-of-responsibleresearch-and-innovation-in-europe-the-morri-indicators-report-d4-3 


\section{Ingeborg Meijer and Wouter van de Klippe}

Owen, R., Macnaghten, P., \& Stilgoe, J. (2012). Responsible research and innovation: From science in society to science for society, with society. Science and Public Policy, 39(6), 751-760.

Pain, R., Whitman, G., \& Milledge, D. (2011). Participatory Action Research Toolkit. http://studentsatthecenterhub.org/wp-content/uploads/2015/04/Engaging-YouthResearchers-partoolkit.pdf

Pandza, K., \& Ellwood, P. (2013). Strategic and ethical foundations for responsible innovation. Research Policy, 42(5), 1112-1125.

Peter, V., Maier, F. J., Mejlgaard, N., Bloch, C., Madsen, E., Griessler, E., Wuketich, M., Meijer, I., Woolley, R., Lindner, R., Bührer, S., Jäger, A., Tsipouri, L., \& Stilgoe, J. (2018). Monitoring the evolution and benefits of responsible research and innovation in Europe: Summarising insights from the MoRRI Project D13. Available at: www.technopolis-group.com/wp-content/uploads/2020/02/Finalreport-\%E2\% 80\%93-Summarising-insights-from-the-MoRRI-project-D13.pdf

Regitz-Zagrosek, V. (2012). Sex and gender differences in health. EMBO Reports, 13(7), 596-603.

Rip, A. (2014). The past and future of RRI. Life Sciences, Society and Policy, $10(1), 17$.

Royal Society. Council. (1985). The Public Understanding of Science. London: The Royal Society.

Smallman, M., Lomme, K., \& Faullimmel, N. (2015). D 2.2. Report on the Analysis of Opportunities, Obstacles and Needs of the Stakeholder Groups in RRI Practices in Europe.

Stilgoe, J., \& Lindner, R. (2015). Analytical Report on the Dimension of Research and Innovation Governance. Available at: https://www.technopolis-group.com/ report/analytical-report-dimension-research-innovation-governance-d2-4-2/

Stilgoe, J., Owen, R., \& Macnaghten, P. (2013). Developing a framework for responsible innovation. Research Policy, 42(9), 1568-1580.

Strand, R., \& Kaiser, M. (2015). Report on Ethical Issues Raised by Emerging Sciences and Technologies. Bergen, Norway: Centre for the Study of the Sciences and the Humanities, University of Bergen.

Talmon-Gros, L., \& Teichler, T. (2015). Analytical report on the dimension of science literacy and scientific education. Available at: http://morri-project.eu/reports/ 2015-04-01-d2.2

Van den Eynden, V., \& Bishop, L. (2014). Incentives and Motivations for Sharing Research Data: A Researcher's Perspective. Colchester, UK: University of Essex, 19, p. 2016. Available at: http://www.knowledge-exchange.info/event/ sowing-the-seed.

Von Schomberg, R. (2013). A vision of responsible research and innovation. In: Owen, R., \& Bessant, J. (eds.) Responsible Innovation: Managing the Responsible Emergence of Science and Innovation in Society. London: John Wiley, pp. 51-74.

Von Schomberg, R., \& Hankins, J. (eds.). (2019). International Handbook on Responsible Innovation: A Global Resource. Cheltenham, UK: Edward Elgar Publishing.

West, C., \& Zimmerman, D. H. (1987). Doing gender. Gender and Society, 1, 125-151.

Wickson, F., \& Carew, A. L. (2014). Quality criteria and indicators for responsible research and innovation: Learning from transdisciplinarity. Journal of Responsible Innovation, 1(3), 254-273. 
Wilsdon, J., Doubleday, R., \& Stirling, A. (2015). Future Directions for Scientific Advice in Europe. Cambridge: Centre for Science and Policy.

Woolley, R., \& Rafols, I. (2016). Development of metrics and indicators for RRI projects, Available at: https://www.technopolis-group.com/wp-content/uploads/ 2017/09/D6.pdf

Wuketich, M., Lang, A., Griessler E., \& Polt, W. (2016). In-depth case studies on the benefits of RRI across the scientific disciplines and industrial sectors. Available at: www.technopolis-group.com/report/depth-case-studies-benefits-rri-acrossscientific-disciplines-industrial-sectors-d5-2/

Wynne, B. (1995). Public understanding of science. In: S. Jasanoff, G. E. Markle, J. C. Peterson, \& T. Pinch (eds.), The Handbook of Science and Technology Studies. London: SAGE Publications, pp. 361-388.

Wynne, B. (2006). Public engagement as a means of restoring public trust in sciencehitting the notes, but missing the music? Community Genetics, 9(3), 211-220. 